\begin{tabular}{|c|c|}
\hline \multirow{3}{*}{ 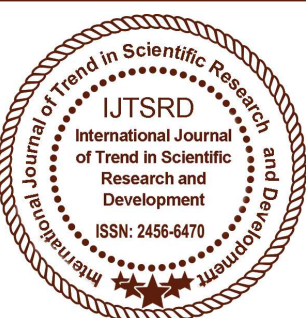 } & $\begin{array}{l}\text { International Journal of Trend in Scientific } \\
\text { Research and Development (IJTSRD) }\end{array}$ \\
\hline & International 0 \\
\hline & ISSN No: 2456 - 6470 | www.ijtsrd.com | Volume - 2 | Issue -4 \\
\hline
\end{tabular}

\title{
Optimization of Scheduling in FMS using Heuristic Approaches: A Case Study
}

\author{
Gaganpreet Kaur ${ }^{1}$, Gayatri Devi ${ }^{2}$, \\ Abhishek Kumar Mishra ${ }^{3}$, Amarnath Kumar ${ }^{3}$, Akarsh Mishra ${ }^{3}$, Aditya Agrahari ${ }^{3}$ \\ ${ }^{1}$ Associate Professor, ${ }^{2}$ Research Scholar, ${ }^{3}$ Student \\ ${ }^{1,3}$ Department of Mechanical Engineering, ABES Engineering College, Ghaziabad, Uttar Pradesh, India \\ ${ }^{2}$ Delhi Technical University (DTU), Shahbad Daulatpur, Main Bawana Road, Delhi, India
}

\section{ABSTRACT}

The flow shop scheduling problems are experiencing a huge eminence in this modern era of industrialization. In the present scenario we find almost every industry to be focusing prominently on the scheduling of the machines working in them, as it is a significant factor in deciding the net productivity. Nowadays almost each and every manufacturing system is involved in following a preplanned schedule of machine operation considering the demand criterion put forth. Flexible manufacturing system being one of them is also following the same path. The largely increasing number of machines and jobs involved in the manufacturing operations has marked a huge complexity in their scheduling. Thus a proper scheduling technique is the only solution which can prove as an aid to the situation. Moving forth in the same direction, this thesis presents a few prominent methods to solve a flow shop scheduling problem. Some major heuristics algorithms are the CDS algorithm, Palmer's algorithm, and NEH algorithm are considered in this paper for finding the most optimized result. A comparison between the heuristics algorithms has also been included in this thesis to make some conclusions upon their individual and cumulative efficiencies. The input parameters given in the problem are considered to be the basis while calculating the make span. To reduce the time involved in the whole process and the chances of human error in the calculations, a java programming code has also been generated.
Keywords: Flexible Manufacturing System, Scheduling, Heuristics, Palmer Heuristics, CDS Heuristics, NEH Heuristics

INTRODUCTION:

Flexible manufacturing systems (FMS) are distinguished by the application of computer systems in replacing the hard automation usually found in transfer lines for the process controlling purposes. The rapid reconfiguration to produce multiple part types is thus enabled to FMS. The setup time is practically eliminated by the use of fixtures and tool magazines. The economic production of a large variety of parts in low volumes is thus permitted by these features only. The additional advantages of features like rapid turnaround, high quality, low inventory costs, and low labour costs are the major reason behind the rapid and heavy adaptation of FMS's in the manufacturing sector. The consistent research works and the high investments in this area are the result of the great potential of FMS as a strategic competitive tool. The system is specified in the design stage in order to achieve the desired performance goals. The main objective of the operation problems is to make decisions regarding the planning, scheduling and control processes of a given FMS. The paper mainly contains a review covering the already published literature on the operation problems of FMS. The first paper was published in this area in the late 1970's. Since then a wide range of research literature has accumulated in this direction. Some surveying works in the literature is also done. 
The analytical models and the scheduling problems are some common perspectives which are reviewed in this paper. Also an attempt has been made to consider the methodological perspectives while focusing on the operation problems. Moreover the review has been brought up to date taking the following viewpoints into consideration:

$>$ Methodology applied in solving the problem

$>$ Applications perspective

$>$ Time horizon consideration

$>$ FMS factors measured

The final section focuses on the further studies and the future researches that are still possible in this area.

Scheduling, on the other hand, is an essential decision making process that has its consistent applications in almost every manufacturing and servicing industry. It focuses on allocating resources to various tasks over the assigned period of time in order to optimize the objective. Different forms are usually taken by the various tasks and resources present in an industry. The resources can be workshop machines, airport runways, construction site crew, working units present in a computing environment, and many more. Similarly the tasks can include various production processes, airport landings and take offs, construction project stages, computer programs execution, etc.

Every task has its own priority level depending upon its starting time and a due date. Most of the time different forms are even taken by the objectives of the problem. Reducing the number of tasks getting finished after their individual due dates can be one of such objectives.

Scheduling has a crucial role to play in the process of decision making in almost every manufacturing and production units along with its application in the information processing environment. Also it has a significant role in the servicing industries as well as in the transportation and distribution systems.

\section{OBJECTIVE}

This research article is all about jobs scheduling in flow shop environment, and is having the following objectives: -

1. Minimizing the make span value obtained by applying various approaches.

2. Obtaining an optimal sequence for all jobs and machines involved in the process which would specify the job processing sequence to be followed to achieve the best results.
3. Measuring and comparing the suitability of the heuristics considered in this flow shop problem.

\section{LITERATURE REVIEW}

Johnson [1] gave the first algorithm for finding the optimum sequence in case of an n-job 2-machine problem.

Palmer [2] gave a method which involved a single iteration in order to come to the desired outcome that is the make span minimization. The slope index calculation is marked as the basis for the job sequencing which are arranged in the decreasing array in order to achieve the optimum sequence through this method. This can further be applied to n-job mmachine scheduling problems.

The extension of the simple Johnson's algorithm was later given by Campbell et al [3]. In contrast to the modified Johnson's algorithms, a huge number of iterations are made in order to come to the desired result. This heuristics approach is generally known as Campbell, Dudek and Smith heuristics (CDS). In this approach the final optimal sequence is obtained from among the various other sequences taken into consideration which is having the minimum make span duration. Also at every iteration the application of Johnson's rule is made.

Gupta [4] proposed a different algorithm for the process of minimizing the final make span of the sequences He further gave a varied method I order to obtain the slope index, which then forms the basis for deciding the job sequence.

Nawaz et al. [5] proposed a method which worked considering its basis to be the processing time of the each job. The jobs are prioritized for the scheduling by giving the job having the maximum processing time a lead over other jobs. Utilizing this concept, he proposed a heuristic for the make span minimization, which further known as the NEH heuristics algorithm.

Nagar et al. [6] suggested a different method for the make span minimization in a flow shop problem. He used two ditinct methods to obtain the result. The two methods are genetic algorithm and branch and bound method.

Nowicki and Smutnicki [7] suggested tabu search for solving the flow-shop scheduling problem. 
Neppalli et al. [8] applied the genetic algorithms approach for solving the two machine scheduling problem with the aim to minimize the make span.

Biskup and Herrmann [9] together proposed a method using due dates as its constraints. The method can be applied for single machine problems. Its objective is to minimize the penalties involved in case the demands are not fulfilled before the due date.

He and Hui [10] gave there contribution in the scheduling of a multi-product and single-stage batch plants on parallel units by utilising the idea of evolutionary agorithim. The genetic algorithm is applied here as the evolutionary algorithm.First the problem is solved which is large in nature using genetic algorithim and then later by using heuristic approach it is finally proposed.

Tseng and Liao [11] worked on a n-job and mmachine flow shop scheduling problem to decrease the total tardiness and earliness. The evolutionary algorithm considered by them is particle swarm optimization technique. To meet the company demand they reduced the weighted earliness and weighted tardiness which they calculated using the particle swarm technique.

$\mathrm{Wu}$ and Zhou [12] founded the required schedule of jobs using a stochastic scheduling approach.For decreasing the lateness of job completion with a single machine it utilizes a stochastic environment.The lateness value of every job from a set of jobs with various due dates allotted to them is found out and then the maximum lateness value is calculated and then that value is minimized.

Mosheiov and Sarig [13] did there work based on the minimizing of the cost factors which detoriates the company's income. The individual cost factors include lateness, tardiness, earliness, latest due date demanded, number of tardy jobs. The model focusses on the point of reference for the scheduling of a sequence of jobs that will minimizethe lateness, due dates, tardiness, and other cost factors.

Cheng and Lin [14]worked on acombination of different methods coprising of Johnson's rules, the introduction of composite jobs and the concept of relocation.As in Johnson's algorithm this technique also considers the two machines problems for finding the flow shop scheduling problem. The reducing of make span time is done as naturally jobs are done with the same idle time as that of the real working machine. This conceptof composite jobs aims at reducing the make span.

Modrak et al. [15] compared the different heuristics algorithms with the output value of the make span time. The heuristics which are used are Gupta's algorithm, Palmer'algorithm, MOD heuristics algorithm, CDS algorithm, NEH algorithm. Since palmer only uses single iteration for the final result on the oher hand NEH used maximum iterations. Though palmer's algorithm was rapid but itmissed accuracy and the results found from palmer's algorithm are not simillar with the best result from alldifferent heuristics algorithm.

Chia and Lee [16] worked on the concept of learning effect for the improvement of a model to decrease the overall completion time. The overall completion time in different flow shop environments is reduced as needed through this method by initiating the idea of learning. By involving different dominance rules and parameters the process speed is accelerated.

Lia et.al [17] proposed a way to obtain the optimal solution by minimizing the overall flow time involved. The composite heuristics models are used in this method for obtaining the total flow time in the flow shop environment. In this method a particular sequence is followed for the processing of jobs. Also the heuristics approach obtained worked well in reducing the total flow time.

\section{PROCESS METHODOLOGY}

Following is the flowchart showing the stepwise procedure that is adopted throughout the tenure of this project in order to get the desired output i.e. the objective of optimizing the scheduling of the FMS taken into consideration and minimizing the total make span time of manufacturing taking place there.

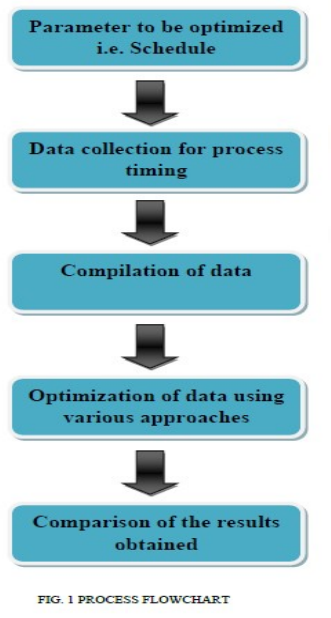




\section{DETAILS OF PROJECT WORK}

I. CDS HEURISTIC APPROACH

METHODOLOGY:

$>$ We have to apply the CDS heuristic approach to the data collected.

$>$ For this purpose, CDS heuristic approach is applied manually.

$>$ Since we have 5 machines in total, so therefore there are total (5-1) i.e. 4 iterations possible.

$>$ Johnson's Algorithm is applied individually to the all-4possible iterations to get a job sequence.

$>$ Now, we have to calculate process time for each of the sequence obtained using the 4 iteration.

$>$ For this purpose, CODING IN JAVA is written.

$>$ Using the Java program, process time for each sequence is determined.

$>$ The sequence which gives the lowest process time is selected as the optimal sequence.

The optimum sequence based on $\mathrm{CDS}$ heuristic method is found to be J4-J2- J1-J3 with minimum process time $58.54 \mathrm{~s}$.

\section{PALMER HEURISTIC APPROACH}

METHODOLOGY:

$>$ We have to apply the Palmer Heuristic approach to the data collected.

$>$ For this purpose an OBJECT ORIENTED CODING IN JAVA is written.

$>$ The data is given as the Input to the program developed.

$>$ The Java program calculates the below mentioned parameters and display them as the output.

- Weights allocated to different machines in order to apply palmer heuristic approach.

- Value calculated on basis of weight allocated to the machines.

- Lower Bound based on each machine.

- Maximum Lower Bound, which is selected for quality calculation.

- Optimal sequence obtained by applying Palmer heuristic approach.

- The process time matrix.

- Process completion time.

- Quality of the solution given by Palmer Heuristic.

Sequence obtained using Palmer Heuristics approach is $\mathrm{J} 4-\mathrm{J} 2-\mathrm{J} 3-\mathrm{J} 1$ with a process completion time of $58.54 \mathrm{~s}$.
The quality of solution given by Palmer Heuristics Method is found to be 0.9558 i.e. the probability that sequence given by this method is optimize is $95.58 \%$.

\section{NEH HEURISTIC APPROACH}

METHODOLOGY:

$>$ We have to apply the NEH heuristic approach to the data collected.

$>$ For this purpose, NEH heuristic approach is applied manually.

$>$ For this purpose, Job Addition Matrix is calculated.

$>$ Since; we have 4 jobs in total, so there are total 4 rows in the matrix calculated.

Now, the jobs are arranged in decreasing or in non increasing order according to the sum associated with each job in the job addition matrix.

The first two jobs are then taken in the sorted sequence and formulated in different combinations.

Now, we have to calculate process time for each of the sequence obtained in the previous step.

For this purpose, Coding in Java is written.

Using the Java program, process time for each sequence is determined.

Out of above combinations, combination with minimum make span is selected for the next step.

Insert the next job from the sequence obtained in. Find out all possible combination of the 3 jobs now and make span time in each of the case is calculated using the same Java coding used in previous step.

$>$ This is continued till a sequence with all 4 jobs is obtained with the least make span time.

$>$ That sequences the optimal sequence given by the NEH heuristic approach.

\section{RESULT \& DISCUSSION}

$>$ Comparisons are done between three algorithms namely CDS Algorithm, Palmer's Algorithm, and NEH Algorithm for minimization of make span in view of our problem.

$>$ By our results we found out that for our 4 job 5 machines problem, the make span obtained is the same i.e. 58.54 for each of the algorithms.

$>$ Also the above make span time is obtained using three job sequences which are, 
International Journal of Trend in Scientific Research and Development (IJTSRD) ISSN: 2456-6470

1. J4-J3- J2-J1 i.e. Spain Coin- Indian 5 coinIndian 10 coin- Peru coin

3. J4-J2- J3-J1 i.e. Spain coin- Indian 10 coinIndian 5 coin- Peru coin

4. J4-J1- J2-J3 i.e. Spain coin- Peru coin- Indian 10 coin- Indian 5 coin

\section{CONCLUSION\& RECOMMENDATION}

Following are the conclusions derived after considering the various approaches in order to fulfill the objective of this research work.

$>$ The different algorithms applied evaluate the following number of sequences:

- Palmer's algorithm:- 1

- CDS algorithm: - 4

- NEH algorithm: - 13

$>\mathrm{NEH}$ Heuristics Algorithm will give more accuracy than CDS Algorithm as it evaluates more number of sequences than the former.

$>$ The maximum lower bound calculated (obtained for machine 5) is 55.96.It means machine 5 alone will take $55.96 \mathrm{sec}$ to process all the 4 jobs neglecting all types of time losses i.e. the earliest at which all jobs can be processed is 55.96

$>$ The optimal time given by various possible optimal sequences is $58.54 \mathrm{sec}$ So-

$>$ Earliest Possible Time $=55.96 \mathrm{sec}$

$>$ Optimal time calculated $=58.54$

$>$ Therefore, quality of $(55.96 / 58.54) * 100=95.58 \%$

$>$ The optimal sequences obtained by various heuristics approaches are-

> Palmer's algorithm:- J4-J2- J3-J1

$>$ CDS algorithm:- J4- J2- J1-J3

$>$ NEH algorithm:-J4-J3- J2-J1, J4-J2-J1-J3 \& J4J1- J2-J3.

\section{FUTURE SCOPE}

All the three algorithms used although analyze many sequences yet they may not be giving all the possible solutions as there are many other algorithms which analyze more sequences to obtain the optimal solution.

$>$ Hence for this flow shop problem there is further more scope for minimizing the make span obtained by these mentioned algorithms.

\section{LIMITATIONS}

$>$ Except the Palmer's algorithm, the CDS-heuristic \& NEH algorithms used for mathematically finding the optimized job sequence for the flow problem were little complex and time consuming.
2. J4-J2- J1-J3 i.e. Spain Coin- Indian 10 coinPeru coin- Indian 5 coin

However java programming came as an efficient aid.

Since, we visited a small scale industry; therefore, we have only small number of jobs. So, it is the reason we may be getting same time by applying all the approaches.

Also, due to less number of jobs, the importance of java programming is not properly depicted.

\section{REFERENCE}

1. Johnson, S. M. (1954), Optimal two- and threestage production schedules with setup times included. Naval Research Logistics, 1, 61-68.

2. Palmer, D. S. (1965), Sequencing jobs through a multi-stage process in the minimum total time - a quick method of obtaining a near optimum, Operations Research Society, 16, 101-107.

3. Campbell, H. G.; Dudek, R. A. and Smith, M. L. (1970), A Heuristic Algorithm for the $\mathrm{n}$ Job, m Machine Sequencing Problem, Management Science, 16-10, 630-637.

4. Gupta, J. N. D. (1971), A functional heuristic algorithm for the flow shop scheduling problem, $22,39-47$.

5. Nawaz, M.; Enscore, E. and Ham, I. (1983), A heuristic algorithm for the $m$ machine, $n$ job flow shop sequence problem, OMEGA, 11, 91-95.

6. "Nagar, A.; Heragu, S. S. and Haddock, J. (1996), A combined branch and bound and genetic algorithm based approach for a flow shopscheduling problem, Annals Operation Research, Springer, 63, 397-414.

7. Nowicki, E. and Smutnicki, C. (1996), A fast tabu search algorithm for the permutation flow-shop problem, European Journal Operation Research, 91, 160-175.

8. Neppalli, V. R.; Chen, C. L. and Gupta, J. N. D. (1996), Genetic algorithms for the two-stage bi criteria flow shop problem. European Journal of Operational Research, 95, 356-373.

9. Biskup, D. and Herrmann, J. (2008), A Singlemachine scheduling against due dates with pastsequence- dependent setup times, European Journal of Operational Research, 19, 587-592.

10. He, Y. and Hui, W. C. (2008), A rule-based genetic algorithm for the scheduling of single- 
stage multi-product batch plants with parallel units, Computers and Chemical Engineering, 32, 3067-3083.

11. Tseng, C. and Liao, C. J. (2008), A discrete particle swarm optimization for lot- streaming flow shop scheduling problem, European Journal of Operational Research, 191, 360-373.

12. Xianyi, W. and Zhou, X. (2008), Stochastic scheduling to minimize expected maximum lateness, European Journal of Operational Research, 190, 103-115.

13. Mosheiov, G. and Sarig, A. (2009), The due-date assignment on uniform machines, European Journal of Operational Research, 193, 49-58.

14. Cheng, T.C.E. and Lin, B. (2009), Johnson's rule, composite jobs and the relocation problem,
European Journal of Operational Research, 192, 1008-1013.

15. Modrak, V.; Semanco, P. and Kulpa, W. (2009), Performance measurement of selected heuristics algorithm for solving scheduling problems European Journal of Operational Research, 34, 158-183.

16. Wu, C. and Lee, W. C. (2009), A note on the total completion time problem in a permutation flow shop with a learning effect, European Journal of Operational Research, 192, 343-347.

17. Li, X.; Wang, Q. and Cheng, W. (2009), Efficient composite heuristics for total flow time minimization in permutation flow shops, Omega, $37,155-164$. 